\title{
Hand bone densitometry: a more sensitive standard for the assessment of early bone damage in rheumatoid arthritis
}

\author{
Glenn Haugeberg, Michael J Green, Philip G Conaghan, Mark Quinn, Richard Wakefield, Susanna \\ M Proudman, Sheena Stewart, Elizabeth Hensor, Paul Emery
}

See end of article for authors' affiliations

Correspondence to: Professor Paul Emery, Academic Unit of Musculoskeletal Disease Chapel Allerton Hospital Chapeltown Road, Leeds, UK; p.emery@leeds.ac.uk

Accepted 14 April 2007

Published Online First

9 May 2007

\begin{abstract}
Objective: To examine the role of hand dual-energy $x$ ray absorptiometry (DEXA) compared with radiography in the assessment of bone involvement in patients with early rheumatoid arthritis (RA) who have active disease.

Methods: The study population $(n=79)$ had RA of $<12$ months' duration and were selected for poor prognostic features. Clinical data and bone mineral density (BMD) data were collected at baseline, 24 and 48 weeks. Hand radiographs were performed at baseline and 48 weeks. Bone damage analyses were performed for the group and individuals using the smallest detectable change (SDC) method.

Results: At baseline, mean disease duration was 8.5 months, erythrocyte sedimentation rate was $34.3 \mathrm{~mm} /$ hour, C-reactive protein was $40.2 \mathrm{mg} / \mathrm{l}$, Health Assessment Questionnaire score was 1.35 and $81 \%$ of patients were positive for rheumatoid factor. Mean $(95 \% \mathrm{CI})$ hand BMD loss was $2.5 \%(-3.5$ to -1.5$)$ at 24 weeks and $2.6 \%(-3.8$ to -1.5$)$ at 48 weeks. Individual hand bone loss exceeding the SDC was seen in $46.8 \%$ at 24 weeks and in $58.2 \%$ at 48 weeks. In the subgroup of 58 patients who had undergone radiography, radiographic joint damage score evaluated by the Sharp-van der Heijde method increased from 4.8 to 10.6 $(p=0.001)$. Individual hand bone loss in this subgroup exceeding the SDC was seen in $50.0 \%$ at 24 weeks and in $56.9 \%$ at 48 weeks, whereas at 48 weeks only $22.4 \%$ had deteriorated in modified Sharp score.

Conclusion: The study results indicate that hand DEXA is a more sensitive tool than radiology (radiographic joint-damage scores), for measuring disease-related bone damage in early RA.
\end{abstract}

I nflammation-related bone damage in rheumatoid arthritis (RA) presents not only as erosions but also as osteoporosis. ${ }^{1}$ Thus, quantitative bone measures have been proposed as outcome measures in early RA. ${ }^{23}$ Currently only radiographic joint damage is well established as an outcome measure for assessment of disease progression and bone damage in both daily clinical practice and clinical trials (commonly using modifications of the Sharp and Larsen scoring systems). ${ }^{45}$ Unfortunately, the presence of definite erosions on radiographs is a feature of established disease ${ }^{1}$ and is observed less frequently in early disease. With aggressive new therapies, rates of erosion progression are lower and therefore a sensitive outcome measure is required to differentiate between modern treatments.

Of the quantitative bone-measurement methods, dual-energy $x$ ray absorptiometry is considered the gold standard. In RA, disease-related bone loss assessed with DEXA, both generalised $^{6}$ and in the hands, ${ }^{7}$ has been shown to occur in the very early phase of the disease process. Hand bone loss detected by DEXA has even been shown to take place in the undifferentiated stage of the disease before RA can be diagnosed clinically. ${ }^{8}$

The widespread availability of DEXA, its good precision and ease of use gives it the potential to be widely applied in clinical practice and clinical trials for assessment at both group and individual levels; however, there is a need to validate DEXA in early RA.

The aim of this study was therefore to examine hand DEXA as an alternative to radiography as a new sensitive outcome measure in the assessment of bone involvement in early RA.

\section{METHODS}

\section{Study population}

Hand DEXA as a potential bone-damage outcome measure in RA was tested in a cohort with early RA who had symmetrical active polyarthritis affecting the metacarpophalangeal joints. Recruited patients were considered to have a poor prognosis by the presence of various outcome predictors in terms of function and radiographic damage (table 1). ${ }^{9}$ All patients, recruited from the Leeds Early Arthritis clinics, had disease duration of $<12$ months and satisfied the 1987 American College of Rheumatology (ACR) criteria. ${ }^{1}$ The study was approved by the ethics committee of Leeds Teaching Hospitals Trust. Detailed data from this study have previously been published without the bone-density results. ${ }^{10}$ In total, 79 of the 82 patients had DEXA scans performed and were included in this study. Selected demographic and clinical variables for this study are displayed in table 2 .

In this study, a conventional treatment strategy was tested against a more aggressive strategy. The patients were randomised to receive either monotherapy with sulfasalazine or combined therapy with methotrexate, ciclosporin A and methylprednisolone by injection into all active joints at study entry and thereafter as indicated by the presence of clinically active joints. ${ }^{10}$ During follow-up, the conventionally treated patients received sulfasalazine with a median daily dose of 3 (range 0 to 3) g and 68.8 (range 0 to 200) mg methylprednisolone intra-articularly, whereas aggressively treated patients received a mean (SD) dose of methotrexate 9.3 (5.0) mg/week and ciclosporin A 3.0 (2.1) mg/kg/day and a total dose of 154.5 (range 0 to 660) $\mathrm{mg}$ methylprednisolone intra-articularly.

Abbreviations: $A C R$, American College of Rheumatology; BMD, bone mineral density; CRP, C-reactive protein; $E S R$, erythrocyte sedimentation rate; DEXA, dual-energy $x$ ray absorptiometry; $\mathrm{HAQ}$, Health Assessment Questionnaire; MCP, metacarpophalangeal; PIP, proximal interphalangeal; RA, rheumatoid arthritis; RANKL, receptor activator of nuclear factor $\kappa B$ ligand; TNF, tumour necrosis factor; SDC, smallest detectable change; SDD, smallest detectable difference 


\begin{tabular}{|c|c|}
\hline Predictive marker & Points \\
\hline Rheumatoid factor titre $>40 \mathrm{mg} / \mathrm{l}$ & 1 \\
\hline Presence of HLA-DR*04 and or DR*01 & 1 \\
\hline Female sex & 1 \\
\hline C-reactive protein $>20 \mathrm{mg} / \mathrm{l}$ & 1 \\
\hline \multicolumn{2}{|l|}{$\mathrm{HAQ}$} \\
\hline Total >7 & 1 \\
\hline Total $>12$ & 2 \\
\hline \multicolumn{2}{|c|}{$\begin{array}{l}\mathrm{HAQ} \text {, Health Assessment Questionnaire; HLA, human leucocyte } \\
\text { antigen. } \\
\text { Patients were placed in the poor prognosis group if the points } \\
\text { totalled } \geqslant 3 \text {. } \\
\text { Score is the raw HAQ measured out of a total of } 24 \text { points. }\end{array}$} \\
\hline
\end{tabular}

Intramuscular methylprednisolone injection was given to patients in either group as considered necessary by the study doctors. None of the patients received oral corticosteroids during the study period. ${ }^{10}$

\section{Bone densitometry}

One technician performed all DEXA scans including scans for reproducibility assessment using the same DEXA equipment (Lunar Expert, Madison, Wisconsin, USA). All DEXA scans of both hands, lumbar spine (L2-4) and right hip were performed according to a standardised procedure for each site. For wholehand DEXA, all hand bones distal from the wrist joint were included in the measurement, as described by Deodhar et al: ${ }^{11}$ the hand is put flat on a table and finger extensions, if present, are straightened out. The long-term spine phantom precision, expressed as percentage coefficient of variation (CV) calculated from daily phantom measurements was $0.80 \%$ for the whole study period. Hand DEXA was performed at baseline and 24 and 48 weeks follow-up, and for lumbar spine and femoral neck at baseline and 48 weeks. For each group, changes in BMD were expressed as mean values with $95 \%$ confidence intervals (CI).

\section{Bone mineral density in vivo short-term variability}

To define cut-off values for the assessment of individual bone changes in the hand, spine and femoral neck, we used CV and the $95 \%$ limits of agreement method of Bland and Altman ${ }^{12}$. Precision expressed according to the Bland and Altman method $^{12}$ gives an absolute and metric estimate of random measurement error, also called smallest detectable difference (SDD). The method for calculating SDD, detecting the limits of agreement, based on duplicate DEXA measures in individuals, has been described in detail previously. ${ }^{13}{ }^{14}$ To identify the smallest detectable change (SDC) within an individual (rather than the SDD between individuals) a slight adjustment is made to the equation so that

SDC $= \pm 1.96 \times$ SD (difference between two measurements)/ $(\sqrt{ } 2 \times \sqrt{k})$,

where $\mathrm{k}$ is the number of readings over which to average the analyses of the trial. ${ }^{15}$

The CV expresses the SD corrected for the mean of paired measurements. CV, expressed as a percentage, was calculated as

$$
\mathrm{CV} \%=(\sqrt{ }((\Sigma(\mathrm{a}-\mathrm{b}) 2) / 2 \mathrm{n})) /((\mathrm{Ma}+\mathrm{Mb}) / 2) \times 100,
$$

where $\mathrm{a}$ and $\mathrm{b}$ are the first and the second measurements, Ma and $\mathrm{Mb}$ are the mean values for the two groups, and $\mathrm{n}$ is the number of paired observations. ${ }^{13}{ }^{14}$ Because a BMD change for two point measurements in time exceeding $2 \sqrt{2 \mathrm{CV}} \%$ is

\begin{tabular}{|c|c|}
\hline \multicolumn{2}{|l|}{ Baseline demographics } \\
\hline Age (years), mean (SD) & $49.7(13.1)$ \\
\hline Female sex, $\mathrm{n}(\%)$ & $47(59.5)$ \\
\hline $\mathrm{BMI}\left(\mathrm{kg} / \mathrm{m}^{2}\right)$, mean $(\mathrm{SD})$ & $26.2(4.7)$ \\
\hline \multicolumn{2}{|l|}{ Baseline disease variables } \\
\hline Disease duration (months), mean (SD) & $8.5(5.8)$ \\
\hline Rheumatoid factor, $\mathrm{n}(\%)$ & $64(81.0)$ \\
\hline CRP (mg/l), mean (range) & $40.2(2$ to 195$)$ \\
\hline ESR (mm/hour), mean (SD) & $34.3(28.2)$ \\
\hline *HAQ score $(0-3)$, mean (SD) & $1.35(0.71)$ \\
\hline Hand BMD $\left(\mathrm{g} / \mathrm{cm}^{2}\right)$, mean (SD) & $0.43(0.07)$ \\
\hline \multicolumn{2}{|l|}{ Mean follow-up disease variables } \\
\hline CRP (mg/l), mean (range) & $24.7(5$ to 141$)$ \\
\hline${ }^{*} \mathrm{HAQ}$ score $(0-3)$, mean (SD) & $0.86(0.61)$ \\
\hline \multicolumn{2}{|c|}{$\begin{array}{l}\text { BMI, body mass index; CRP, C-reactive protein; ESR, } \\
\text { erythrocyte sedimentation rate; } H A Q \text {, Health Assessment } \\
\text { Questionnaire. } \\
\text { *HAQ score from } 0 \text { (no disability) to } 3 \text { (severe disability). }\end{array}$} \\
\hline
\end{tabular}

considered a significant change with $95 \%$ confidence expressed as percentage change, ${ }^{16}$ these values were also computed. The percentage $\mathrm{CV}$ and the percentage detection limit described by Gluer et al, termed the least significant change, are shown for individuals in table $3 .{ }^{17}$

The variability of hand DEXA BMD measurements for mean of both hands and the right hand were calculated by using baseline and 12 weeks follow-up data in 81 healthy volunteers (table 3 ). We applied the variability results from the mean of both hands to define true individual hand bone loss in the examined RA population (table 3). No significant change in mean hand BMD was seen $(+0.13 \%, 95 \%$ CI -0.13 to 0.39 , $\mathrm{p}=0.30$ ) between the two time points, thus indicating that no significant biological change had occurred over the 12 weeks. For the right femoral neck and lumbar spine (L2-4), variability in DEXA BMD was calculated from duplicate BMD measurements performed on the same day in 48 healthy volunteers. These variability results were used to identify individual patients in the RA cohort with true bone loss in the right femoral neck and L2-4 spine.

\section{Radiographic scoring}

The radiographs of both hands and feet at baseline and 48 weeks were independently scored according to the van der Heijde modification of the Sharp method (Sharp-van der Heijde; scale $0-448),{ }^{18}$ by two trained observers who were unaware of the identity and treatment status of the patients. The radiographs were scored as pairs in chronological order, and the means of the two observers' scores for erosions, jointspace narrowing and total damage were recorded. The calculation of the specific SDD for the Sharp-van der Heijde method applied to patients with radiographs in the study population has previously been published and described in detail. ${ }^{19}$ In summary, this SDD calculation was based on readings of 52 pairs of films from the study population performed by two assessors reading films in a paired chronological order. Lassere reported SDD of 11.0 points for these patients. ${ }^{19}$ By applying the modification to the Bland and Altman 95\% limits of agreement method, ${ }^{12}$ which provides an absolute and metric estimate of random measurement error, the specific SDC for our patients was calculated to be 7.8 units. $^{15}$

\section{RESULTS}

The demographic and disease characteristics of the study cohort are shown in table 2 . 
Table 3 Reproducibility of bone mineral density measurements in the hands, right femoral neck and L2-4 spine

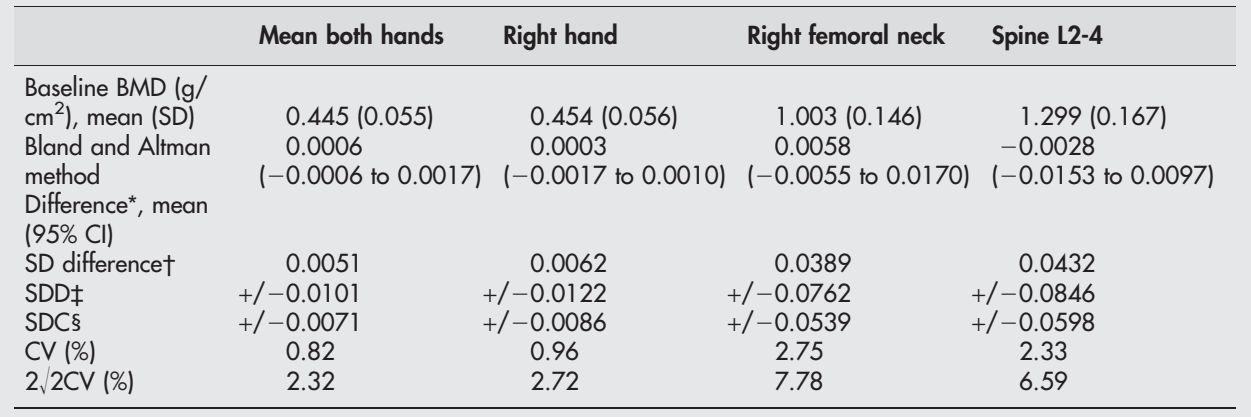

$B M D$, bone mineral density; $C V$, coefficient of variation; mean difference, mean of the difference between the first and the second BMD measurement; SDC, smallest detectable change $\left(\mathrm{g} / \mathrm{cm}^{2}\right)$; SDD, smallest detectable difference $\left(\mathrm{g} / \mathrm{cm}^{2}\right)$; $S D_{\text {difference, }} S D$ of the difference between the first and the second $B M D$ measurement.

*Systematic bias.

†Random measurement error.

‡ 95\% Limits of agreement between individuals (assuming no systematic bias).

$\S \sim 95 \%$ Limits of agreement within individuals (assuming no systematic bias).

\section{Bone density change at group and individual level}

Mean (SD) baseline BMD $\left(\mathrm{g} / \mathrm{cm}^{2}\right)$ was $0.43(0.07)$ in the hand, $0.98(0.15)$ in the right femoral neck, and $1.19(0.16)$ in the lumbar spine (L2-4).

At 24 weeks, mean (95\% CI) hand BMD was reduced by $2.5 \%$ $(-3.5$ to -1.5$)$ and at 48 weeks by $2.6(-3.8$ to -1.5$)$. Hand bone loss exceeding the SDC was seen at 24 weeks in 37 $(46.8 \%)$ and at 48 weeks in $46(58.2 \%)$ patients.

After 48 weeks, mean (95\% CI) BMD reduction was $0.7 \%$ $(-1.9$ to 0.5$)$ in the right femoral neck and $1.9 \%(-3.1$ to -0.8$)$ in the L2-4 lumbar spine. At 48 weeks, $15.4 \%$ (12 of 78) patients had lost BMD exceeding the SDC in the femoral neck, and $21.5 \%$ ( 17 of 79 ) had lost it in the lumbar spine.

\section{Bone density and treatment group}

No significant differences was seen at baseline for mean (SD) BMD between conventionally and aggressively treated patients at the hands $(0.43(0.07)$ vs. $0.42(0.06), p=0.52)$, the right femoral neck $(0.96(0.13)$ vs $1.00(0.16), p=0.20)$ and the lumbar spine L2-4 ( 1.19 (0.15) vs 1.19 (0.15), p =0.84). During the 48-week follow-up, no significant differences in mean bone loss were found between the conventionally and the aggressively treated patients for the hand $(2.68 \%$ vs $2.58 \%, \mathrm{p}=0.88)$, right femoral neck $(0.73 \%$ vs $0.64 \%, \mathrm{p}=0.96)$ or lumbar spine L2-4 (2.13\% vs $1.70 \%, p=0.71)$.

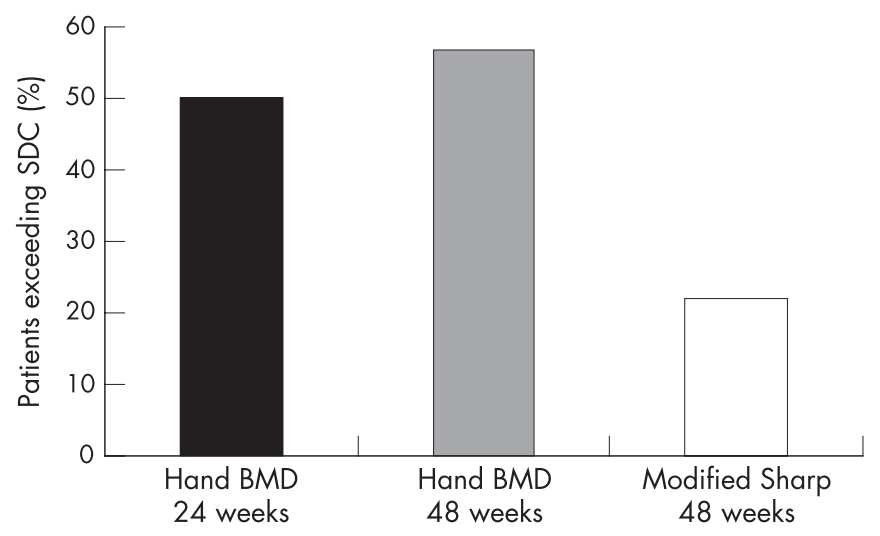

Figure 1 Percentage of 58 patients with rheumatoid arthritis followed up for 48 weeks with bone mineral density (BMD) loss of both hands and deterioration in modified Sharp score (hands and feet) exceeding the SDC $95 \%$ detection limit for the method.

\section{Radiographic progression}

In a subgroup of 58 patients with hand DEXA, hand and foot radiographs from both baseline and 48 weeks were also obtainable, assessed with the modified Sharp score. In these patients, a significant $(\mathrm{p}=0.001)$ increase in mean modified Sharp score from 4.8 (range 0 to 32 ) to 10.6 (0 to 74.5 ) was found. By applying the SDC method with the cut-off values for the modified Sharp score calculated from the examined patient cohort ( 7.8 units, modified from Lassere et al ${ }^{19}$ ) only $22 \%$ ( 13 of 79) of the patients had deterioration in radiographic joint damage exceeding the SDC 95\% detection limit for this method (fig 1). In contrast, at 24 weeks, 50\% (29 of 58) of the patients had hand DEXA BMD loss exceeding the SDC and at 48 weeks $57 \%$ (33 of 79 ) had hand DEXA BMD loss exceeding the SDC (fig 1).

\section{Comparison between bone mineral density and radiographic damage}

The Pearson correlation coefficient between decrease in hand BMD and deterioration in radiographic damage was -0.35 $(p=0.007)$ compared with modified Sharp score, -0.43 $(\mathrm{p}=0.001) \quad$ compared with joint-space narrowing, and $-0.21(p=0.12)$ compared with erosions. Pearson correlation coefficient between joint-space narrowing and erosions was $0.64(p<0.001)$. The mean relative rate of 48 -week change for the hand DEXA method was doubled $(2.8 \%)$ compared with the modified Sharp score $(1.3 \%)$. On the individual level, only six of the 13 patients who deteriorated in modified Sharp score had a "true" hand BMD loss, whereas only 6 of the 33 patients who had a "true" hand BMD loss deteriorated in modified Sharp score $($ McNemar test $\mathrm{p}=0.001)$.

\section{DISCUSSION}

The results from this study provide evidence that hand DEXA is a more sensitive outcome measure than radiographic joint damage scores, particularly for bone damage in early RA. At 24 weeks, mean hand BMD was reduced by $2.5 \%$, and on the individual level approximately half $(47 \%)$ of the patients had lost hand BMD exceeding the measurement error. In the subgroup of 58 patients with both hand radiographs and DEXA, on the individual level only $22 \%$ of the RA patients had deteriorated in modified Sharp score after 48 weeks whereas $50 \%$ had already lost significant hand BMD after 24 weeks. This makes hand DEXA a highly sensitive tool to detect bone damage, especially in the early stage of the disease, a crucial time for assessing new treatments. 
Although bone loss in RA occurs both as periarticular (eg assessed at hand) and generalised osteoporosis (eg assessed in the spine or hip), measures of periarticular osteoporosis are more suitable for assessment of bone damage than measures of generalised osteoporosis, for several reasons. Bone loss in early RA occurs more rapidly in the hand than the hip and spine. ${ }^{8}$ Furthermore, the rate of hand bone loss, as shown in our study, is higher than that in the spine and hip, and the precision of DEXA is also superior at the hand compared with the hip and spine (table 3). By applying our precision data, the advantage of hand DEXA using mean values of both hands compared with hip and spine becomes obvious: in the hands, a bone loss of only $2.3 \%$ is required to identify patients with individual bone loss, whereas in the femoral neck and lumbar spine, bone losses as high as $7.8 \%$ and $6.6 \%$, respectively, are required.

Theoretically, the best site to measure periarticular bone loss in RA would be around the finger joints, particularly the metacarpophalangeal (MCP) joints and proximal interphalangeal (PIP) joints. Our choice to use DEXA of the whole hand instead of the finger joints to assess periarticular bone loss in patients with RA was based on the literature and our own reproducibility tests comparing the two principal measurement strategies. DEXA of the whole hand compared with local measures around single joints is more feasible, ${ }^{20}$ has better precision $^{20}$ and also reflects the whole inflammatory disease process taking place in all affected joints in the hands. In the study by Alenfeld et al, precision (CV) for DEXA of the whole hand was $0.9 \%$, but for periarticular regions at the MCP joints of the second to fifth fingers, precision was poorer, ranging from $2.7 \%$ to $3.2 \%{ }^{20}$ In our own analyses, we also found poorer precision for DEXA measurements around finger joints compared with whole hand measurements. Furthermore, no differences were found for percentage bone loss between DEXA measurements in the finger joints and whole hand (unpublished data).

In contrast to DEXA, the reproducibility for $x$ ray scoring methods may be more dependent on the reader and on the study population. For radiographic scoring systems, it has been shown that $x$ rays even of early RA are scored with great variability, even when scoring is performed by the same reader. ${ }^{21}$ In the study by Lassere et al, assessing reproducibility for the Sharp-van der Heijde method (range 0 to 448), SDD ranged from 4.7 to 15.5 units and was dependent on the source population used for SDD calculation, patient disease activity and the readers. ${ }^{19}$

\section{Limitations}

Direct comparison of the measurement error of the two methods in our study is restricted by several limitations. There is a difference in how the duplicate measures are obtained for the SDC calculation for the two methods. Calculation of the DEXA SDC/SDD is recommended to be based on duplicate measures of scans performed on the same day or some days apart, ${ }^{13}{ }^{14}$ which is a point estimate of the measurement error. For radiographic scoring methods including the Sharp-van der Heijde method, the SDC calculation for practical purposes is based on readings from baseline and follow-up films in each individual, and it is recommended they be read in a paired chronological order by two assessors. Thus, the SDC for DEXA is based on intra-reader reproducibility, whereas the SDC for the radiographic measurements is based on inter-reader reliability. However, this difference in the methods used to assess agreement reflects the intrinsic difference in the practical measurement methods between DEXA and radiographic scoring. Thus, the increased sensitivity of DEXA is a product of the precision of the method. The face validity of the hand DEXA method also needs to be addressed.
The fact that the patients were enriched for features predicting more radiographic damage means that differences between DEXA and radiographic damage were, if anything, underestimated.

Although erosions are pathognomonic for bone damage in $\mathrm{RA}$, bone loss over time is also seen in healthy people, especially in postmenopausal women. However, for short study periods, this does not seem to be a significant problem. In our healthy controls used for assessing the precision of hand DEXA, no bone loss was seen in the hand when the duplicate measurements performed 3 months apart were compared. Joint destruction is also more directly related to patient physical function and health status than is osteoporosis, as bone loss in itself is a symptom-free feature leading to no structural changes on the joint surface. ${ }^{522}$

Radiographic joint damage using the Sharp score (scoring both erosions and joint-space narrowing) has been shown to increase linearly over time. ${ }^{5}$ In contrast, the rate of hand bone loss measured by DEXA has been shown to decrease after the first 2-3 years of disease duration, ${ }^{72}$ which makes DEXA of the hands less usable in the later and established stages of the disease. However, the same pattern is also found for the Larsen score, which only scores erosions. ${ }^{24}$ In a 10 year follow-up study of early RA (disease duration $<12$ months). Linqvist et al showed that radiographic progression using the Larsen score was most rapid during the first 2 years, and that $75 \%$ of all damage occurred during the first 5 years of disease. ${ }^{24}$

In comparison with DEXA, other modern imaging modalities, such as $\mathrm{MRI}^{25}{ }^{26}$ and ultrasonography ${ }^{27}$ are not suitable for assessment of bone mass. They are superior to radiographs in detecting erosions at an early stage of the disease and assessing soft-tissue abnormalities and synovitis; however, they are currently often not as widely available as DEXA, and there is a lack of standardisation for general clinical use in early RA.

To be a clinically relevant outcome measure in early RA, and eventually an alternative to radiographic scoring methods in the early stage of the disease, bone loss should be a direct consequence of the inflammatory disease process and be related to joint erosions. Substantial data from both animal and clinical studies support the theory that the osteoclast cells play a pivotal role in the development of both osteoporosis and erosions in RA. This common cellular mechanism may be explained by activation of the receptor activator of nuclear factor kB ligand (RANKL), which is a key factor in the activation and differentiation of osteoclasts by binding to its receptor RANK on premature osteoclasts. ${ }^{28}{ }^{29}$ Tumour necrosis factor (TNF) $-\alpha$ and interleukin-1, which play a pivotal role in the pathogenesis of synovitis in RA, have been found to be regulators of osteoclastic bone resorbtion by upregulating RANK. ${ }^{30}{ }^{31}$ Studies in human TNF-transgenic (hTNFtg) mice have shown an increase in both joint destruction and generalised bone loss compared with wild-type mice. ${ }^{28}{ }^{32}$ The concept of osteoclast inhibition has recently been confirmed in humans. ${ }^{33}$

In the present study, only patients with RA with poor prognosis were included. Thus, the results are of limited value understanding bone loss in patients with less poor prognostic disease. Further studies in different patient cohorts are therefore warranted to examine the generalisability of the method.

\section{CONCLUSION}

Hand DEXA bone density is more sensitive than conventional hand radiographic joint damage scores in detecting bone damage in early RA. To consolidate the role of hand DEXA as an outcome measure in early RA, we recommend hand DEXA to be included as an outcome measure in both observational and clinical trials, particularly in studies testing the effects of potent disease-modifying treatments. 


\section{Authors' affiliations}

Glenn Haugeberg, Department of Rheumatology Sørlandet Hospital,

Kristiansand, Norway

Glenn Haugeberg, Norwegian University of Science and Technology

(NTNU), Trondheim, Norway

Michael J Green, Philip G Conaghan, Mark Quinn, Richard Wakefield,

Sheena Stewart, Elizabeth Hensor, Paul Emery, Academic Unit of

Musculoskeletal Disease, University of Leeds, Leeds, UK

Susanna M Proudman, Rheumatology Unit, Royal Adelaide Hospital,

North Terrace Adelaide, 5000, Australia

\section{REFERENCES}

1 Arnett FC, Edworthy SM, Bloch DA, McShane DJ, Fries JF, Cooper NS, et al. The American Rheumatism Association 1987 revised criteria for the classification of rheumatoid arthritis. Arthritis Rheum 1988:31:315-24.

2 Haugeberg G, Emery P. Value of dual-energy $x$-ray absorptiometry as a diagnostic and assessment tool in early rheumatoid arthritis. Rheum Dis Clin North Am 2005:31:715-28.

3 Green MJ, Deodhar AA. Bone changes in early rheumatoid arthritis. Best Pract Res Clin Rheumatol 2001;15:105-23.

4 van der Heijde DM. Radiographic imaging: the 'gold standard' for assessment of disease progression in rheumatoid arthritis. Rheumatology 2000;39/Suppl 1):9-16.

5 Drossaers-Bakker KW, de Buck M, Van Zeben D, Zwinderman AH Breedveld FC, Hazes JM. Long-term course and outcome of functional capacity in rheumatoid arthritis: the effect of disease activity and radiologic damage over time. Arthritis Rheum 1999;42:1854-60.

6 Gough AK, Lilley J, Eyre S, Holder RL, Emery P. Generalised bone loss in patients with early rheumatoid arthritis. Lancet 1994;344:23-7.

7 Deodhar AA, Brabyn J, Jones PW, Davis MJ, Woolf AD. Longitudinal study of hand bone densitometry in rheumatoid arthritis. Arthritis Rheum 1995;38:1204-10.

8 Haugeberg G, Green MJ, Quinn MA, Marzo-Ortega H, Proudman S, Karim Z, et al. Hand bone loss in early undifferentiated arthritis: evaluating bone mineral density loss before the development of rheumatoid arthritis. Ann Rheum Dis 2006;65:736-40

9 Emery P. The Dunlop-Dottridge Lecture: prognosis in inflammatory arthritis: the value of HLA genotyping and the oncological analogy. J Rheumatol 1997;24:1436-42.

10 Proudman SM, Conaghan PG, Richardson C, Griffiths B, Green MJ, McGonagle D, et al. Treatment of poor-prognosis early rheumatoid arthritis. A randomized study of treatment with methotrexate, cyclosporin $A$, and intraarticular corticosteroids compared with sulfasalazine alone. Arthritis Rheum 2000:43:1809-19.

11 Deodhar AA, Brabyn J, Jones PW, Davis MJ, Woolf AD. Measurement of hand bone mineral content by dual energy $x$-ray absorptiometry: development of the method, and its application in normal volunteers and in patients with rheumatoid arthritis. Ann Rheum Dis 1994;53:685-90.

12 Bland JM, Altman DG. Statistical methods for assessing agreement between two methods of clinical measurement. Lancet 1986;1:307-10.

13 Lodder MC, Lems WF, Ader HJ, Marthinsen AE, van Coeverden SC, Lips P, et al. Reproducibility of bone mineral density measurement in daily practice. Ann Rheum Dis 2004;63:285-9.

14 Ravaud P, Reny JL, Giraudeau B, Porcher R, Dougados M, Roux C. Individual smallest detectable difference in bone mineral density measurements. J Bone Miner Res 1999;14:1449-56.
15 Bruynesteyn K, Boers M, Kostense P, Van Der LS, Van Der HD. Deciding on progression of joint damage in paired films of individual patients: smallest detectable difference or change. Ann Rheum Dis 2005;64:179-82.

16 Genant HK, Block JE, Steiger P, Glueer CC, Ettinger B, Harris ST. Appropriate use of bone densitometry. Radiology 1989;170:817-22.

17 Gluer CC. Monitoring skeletal changes by radiological techniques. J Bone Miner Res 1999;14:1952-62.

18 Van Der HD. How to read radiographs according to the Sharp/van der Heijde method. J Rheumatol 1999:26:743-5.

19 Lassere MN, van der Heijde D, Johnson K, Bruynesteyn K, Molenaar E, Boonen A, et al. Robustness and generalizability of smallest detectable difference in radiological progression. J Rheumatol 2001;28:911-13.

20 Alenfeld FE, Diessel E, Brezger M, Sieper J, Felsenberg D, Braun J. Detailed analysesin periarticular osteoporosis in rheumatoid arthritis. Osteoporos Int 2000;11:400-7.

21 Paulus HE, Oh M, Sharp JT, Gold RH, Wong WK, Park GS, et al. Correlation of single time-point damage scores with observed progression of radiographic damage during the first 6 years of rheumatoid arthritis. J Rheumatol 2003:30:705-13.

22 van der Heijde D. Radiographic progression in rheumatoid arthritis: Does it reflect outcome? Does it reflect treatment? Ann Rheum Dis 2001;60(Suppl III):47-50.

23 Deodhar AA, Brabyn J, Pande I, Scott DL, Woolf AD. Hand bone densitometry in rheumatoid arthritis, a five year longitudinal study: an outcome measure and a prognostic marker. Ann Rheum Dis 2003;62:767-70.

24 Lindqvist $\mathbf{E}$, Jonsson K, Saxne T, Eberhardt K. Course of radiographic damage over 10 years in a cohort with early rheumatoid arthritis. Ann Rheum Dis 2003;62:611-16.

25 Conaghan PG, O'Connor P, McGonagle D, Astin P, Wakefield RJ, Gibbon WW et al. Elucidation of the relationship between synovitis and bone damage: a randomized magnetic resonance imaging study of individual joints in patients with early rheumatoid arthritis. Arthritis Rheum 2003;48:64-71

26 McQueen F, Lassere M, Edmonds J, Conaghan P, Peterfy C, Bird P, et al. OMERACT Rheumatoid Arthritis Magnetic Resonance Imaging Studies. Summary of OMERACT 6 MR Imaging Module. J Rheumatol 2003;30:1387-92.

27 Wakefield RJ, Gibbon WW, Conaghan PG, O'Connor P, McGonagle D, Pease $\mathrm{C}$, et al. The value of sonography in the detection of bone erosions in patients with rheumatoid arthritis: a comparison with conventional radiography. Arthritis Rheum 2000;43:2762-70.

28 Redlich K, Hayer S, Maier A, Dunstan CR, Tohidast-Akrad M, Lang S, et al Tumor necrosis factor alpha-mediated joint destruction is inhibited by targeting osteoclasts with osteoprotegerin. Arthritis Rheum 2002;46:785-92.

29 Gravallese EM, Goldring SR. Cellular mechanisms and the role of cytokines in bone erosions in rheumatoid arthritis. Arthritis Rheum 2000:43:2143-51.

30 Hofbauer LC, Lacey DL, Dunstan CR, Spelsberg TC, Riggs BL, Khosla S. Interleukin-1 beta and tumor necrosis factor-alpha, but not interleukin-6, stimulate osteoprotegerin ligand gene expression in human osteoblastic cells. Bone 1999:25:255-9.

31 Nakashima T, Kobayashi Y, Yamasaki S, Kawakami A, Eguchi K, Sasaki H, ef al. Protein expression and functional difference of membrane-bound and soluble receptor activator of NF-kappaB ligand: modulation of the expression by osteotropic factors and cytokines. Biochem Biophys Res Commun 2000;275:768-75.

32 Schett G, Redlich K, Hayer S, Zwerina J, Bolon B, Dunstan C, et al. Osteoprotegerin protects against generalized bone loss in tumor necrosis factortransgenic mice. Arthritis Rheum 2003;48:2042-51.

33 Jarrett SJ, Conaghan PG, Sloan VS, Papanastasiou P, Ortmann CE, $\mathrm{O}^{\prime}$ Connor PJ, et al. Preliminary evidence for a structural benefit of the new bisphosphonate zoledronic acid in early rheumatoid arthritis. Arthritis Rheum 2006;54:1410-14. 